\title{
Continuity in diabetes care
}

\author{
Sanjay Kalra, Yashdeep Gupta ${ }^{1}$, Ashok Kumar Das ${ }^{2}$ \\ Department of Endocrinology, Bharti Hospital, Karnal, Haryana, ${ }^{1}$ Department of Medicine, Government Medical College and Hospital, \\ Chandigarh, ${ }^{2}$ Pondicherry Institute of Medical Sciences, Puducherry, India
}

The terms compliance, adherence, concordance, and persistence, all indicate different aspects of a person's relationship with his or her prescribed therapy. All these concepts have been discussed in detail, and their pros and cons debated earlier. ${ }^{[1]}$ Irrespective of their different connotations, they all portray a drug-centered picture of treatment. Though important in their own right, these words totally ignore other, equally relevant, aspects of chronic health care, such as the patient - provider relationship. The phrase "therapeutic alliance" tries to encompass this facet of management, ${ }^{[2]}$ but does not succeed fully.

Diabetes care is a multifaceted, long lasting, bidirectional relationship between a person with diabetes and his or her health care provider. This relationship involves much more than a single prescription. Its addresses, and is influenced by, various biological, psychological, social, and environmental factors. ${ }^{[3]}$

We hereby propose a new term, "continuity", which can be used in chronic disease management in general, and diabetes care in specific. Continuity can be defined as a state in which the person with diabetes continues to seek care from a particular health care provider, or a health care organization. A discontinuity implies a break in this relationship, with the person in diabetes choosing to, or being compelled to, stops taking care from his or her provider or organization. Discontinuity may be absolute or partial, and temporary or indefinite. Absolute

\begin{tabular}{|l|l|}
\hline \multicolumn{2}{|c|}{ Access this article online } \\
\hline Quick Response Code: & Website: \\
\hline & www.joshd.net \\
\hline & \\
\hline
\end{tabular}

discontinuity implies complete cessation of a professional relationship with the provider or organization. Partial discontinuity connotes that for certain but not all, services, whether diagnostic or therapeutic, the person with diabetes has changed his or her provider.

As we try to enhance the quality of diabetes care in various ways, we need to focus on the concept of continuity. Diabetes care professionals should aim to expand continuity, while focusing on the reason which can cause a discontinuity.

\section{REASONS FOR DISCONTINUITY}

Continuity of care, in diabetes, however, is easier said than done. Discontinuity is often by choice [Table 1]. Being a chronic illness, which may not necessarily be acutely symptomatic, diabetes allows people who live with it to search for information, and take decisions

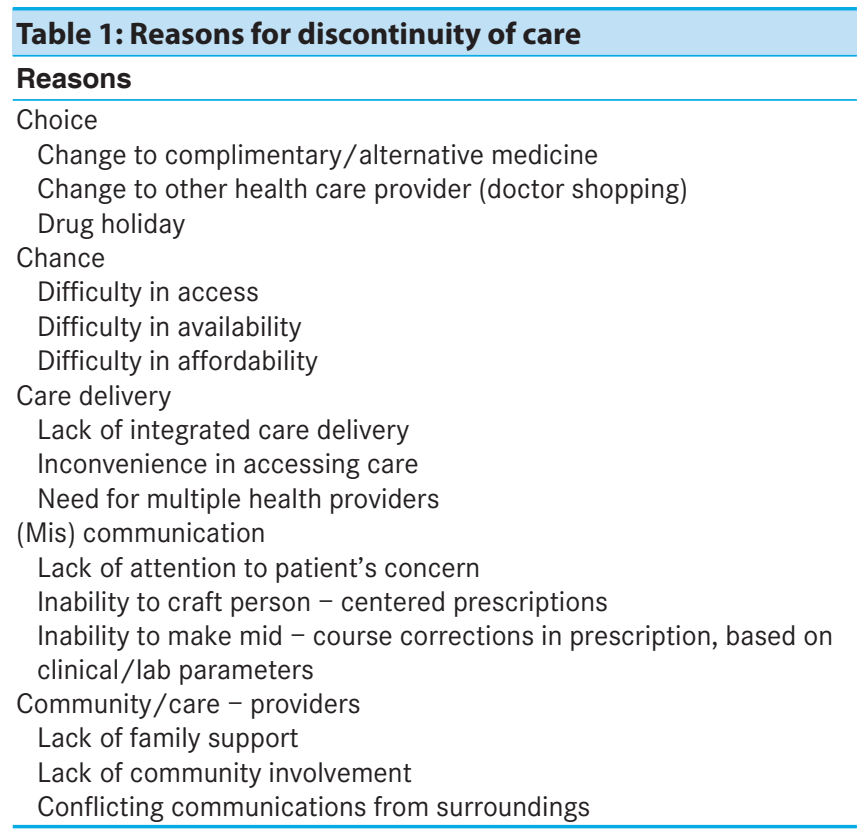

Corresponding Author: Dr. Sanjay Kalra, Bharti Hospital, Kunjpura Road, Karnal - 132 001, Haryana, India.

E-mail: brideknl@gmail.com 
without the help of health care professionals. Thus, such people may choose to drop out of a particular, or all, health care providers' network. ${ }^{[4]}$ They may choose to use complementary and alternative medicine, a situation often seen when insulin is prescribed to an unwilling person.

Discontinuity may also be by chance. In some developing countries, persons with diabetes may have to travel long distances in order to obtain drug and ancillary supplies. In other situations, prescribed drugs may be out of stock for various reasons. Discontinuity of therapy, therefore, may be due to lack of accessibility of treatment. ${ }^{[5]}$

Another reason for the discontinuity of care, apart from choice and chance, is (mis) communication. An unpleasant encounter with a health care professional, his or her inability to explain the cause or course of symptoms or side effects, unwillingness to craft a person-friendly regime suited to individual needs and preferences, or difficulty in effecting mid-course corrections if an initial regime has not provided desired results, fall in this category.

Lack of clinical care can also lead to discontinuity. The lack of integrated health care services providing multidisciplinary care may lead to discontinuation from the diabetes clinic. The need to consult more than one health care provider in gestational diabetes mellitus, or in diabetic retinopathy or nephropathy, for example, may prevent the ill person from seeking diabetes care as regularly as is required.

\section{MAXIMIZING CONTINUITY}

The very reasons which contribute to discontinuity must be addressed in order to maximize continuity in diabetes [Table 2].

Care delivery must be made more efficient, by streamlining workflow processes. Diabetes care providers can be sensitized to the need for delivering comfortable and compassionate care. This can be done by training them in soft skills.

At the same time, core clinical care should not be ignored. A catalog approach can be followed, in which interactive conversation takes place to help decide upon a mutually agreed path of management. Providing a choice to the person with diabetes, e.g., in getting an investigation at a later date, helps retain patients and promotes continuity. This is achieved by ensuring bidirectional communication, in real time, in an open, nonjudgmental manner. The

\begin{tabular}{l}
\hline Table 2: Maximizing continuity in diabetes care \\
\hline Reasons \\
\hline Care delivery \\
Efficient \\
Comfortable \\
Compassionate \\
Communication \\
Bidirectional \\
Real-time \\
Open, nonjudgmental \\
Clinical care \\
By choice, not by coercion \\
Catalogue approach \\
Shared decision-making \\
Corrective measures \\
Lifestyle \\
Life stage \\
Environmental factors \\
Caregiver \\
Active involvement \\
Education \\
Empowerment
\end{tabular}

diabetes care provider should be able to listen as well as speak, and watch for cues which indicate biomedical or psychosocial issues to be addressed.

The diabetes consultation is not a one-time affair. It is an infinite relationship between the person with diabetes and his or her care provider. It stands to reason that such a relation has to be dynamic, with the capacity to adapt. Flexibility, as required by changes in lifestyle, life stage, or altered environmental factors, is a hallmark of a procontinuity diabetes care system.

Such system also draws upon the support of caregivers, including the family, friends, colleagues and community.

\section{CONCLUSION}

The concept of continuity is an umbrella approach which goes beyond conventional use of the term compliance, adherence, concordance, and persistence.

Continuity projects a balanced, person-centered definition of the relationship between the person with diabetes and his or her health care provider. It focuses upon the larger picture and includes all stakeholders such as health care organizations, delivery systems, and the family or community in its ambit. Continuity of treatment helps reduce health care costs, ${ }^{[4]}$ and improves therapeutic outcomes.

As diabetes care evolves and expands, measuring and maximizing continuity should become a major challenge for us. 


\section{REFERENCES}

1. Lutfey KE, Wishner WJ. Beyond "compliance" is "adherence". Improving the prospect of diabetes care. Diabetes Care 1999;22:635-9.

2. Reach G. Observance in diabetes: From therapeutic education to therapeutic alliance. Ann Med Interne (Paris) 2003;154:117-20.

3. Kalra S, Balhara YS, Das AK. The bio-psycho-social model and the American Diabetes Association European Association for the Study of Diabetes position statement on management of hyperglycemia. J Soc Health Diabetes 2013;1:53-5.
4. Ascher-Svanum H, Lage MJ, Perez-Nieves M, Reaney MD, Lorraine J, Rodriguez A, et al. Early discontinuation and restart of insulin in the treatment of type 2 diabetes mellitus. Diabetes Ther 2014;5:225-42.

5. Chinenye S, Ogbera AO, Kalra S. Patient-centered care in diabetology: Sub-Saharan African perspectives. Afr J Diabetes Med 2014;22:5-8.

How to cite this article: Kalra S, Gupta Y, Das AK. Continuity in diabetes care. J Soc Health Diabetes 2015;3:67-9.

Source of Support: Nil. Conflict of Interest: None declared. 